\title{
Atrial dissociation
}

\author{
Abdul Hafiz Khan \\ From the Department of Medicine (Clinical Cardiology), \\ Royal Postgraduate Medical School, Hammersmith Hospital, London
}

A case of atrial dissociation is presented with a review of some of the previously reported cases. The proposed mechanism of atrial dissociation is briefly described. Atrial infarction with ventricular myocardial infarction may have been the cause of observed atrial dissociation.

Atrial dissociation is characterized by two sets of independent $\mathbf{P}$ waves on the electrocardiogram and two atrial beats independent of each other. One atrium is under the control of the dominant pacemaker (sinus node) which also controls the ventricles, while the other atrium or part of the other atrium is under the control of an ectopic pacemaker, resulting in unilateral ectopic atrial rhythm, because the two pacemakers do not interfere with each other. The unilateral ectopic rhythm may be in the form of slow ectopic atrial rhythm, atrial tachycardia, flutter, or fibrillation. The unilateral ectopic rhythm never conducts to the ventricles.

Atrial dissociation has been observed in the exposed heart of the experimental animal. Condorelli (I929) produced atrial dissociation by ligating the left anterior atrial artery, which resulted in sinus rhythm controlling the right atrium, and fibrillation in the left atrium. This phenomenon has been seen in the human heart (Scherf and Cohen, 1964). The following patient is reported in order to illustrate atrial dissociation with a review of some of the cases already reported.

\section{Case report}

The patient was a 78-year-old woman who was admitted with a history of retrosternal chest pain radiating to the left arm of a few hours' duration before admission, after which she became stuperose and incoherent.

She had been taking digoxin $0.25 \mathrm{mg}$ daily for the past 5 years. Physical examination revealed an elderly woman who was pale and sweating with a pulse rate of 75 a minute. The blood pressure was $90 \mathrm{mmHg}$ systolic, and the jugular venous pressure $5 \mathrm{~cm}$ above the sternal angle. The respiratory rate was 20 a minute and difficulty in breathing was evident. Peripheral cyanosis was present, the cardiac impulse was palpable in the sixth intercostal space in the anterior axillary line. The rhythm was regular, the heart sounds were distant, and no murmurs could be heard. A loud early diastolic gallop was audible. Bilateral basal crepitations were present. Abdominal examination was normal and neurological examination did not reveal any localizing signs.

A diagnosis of acute myocardial infarction was made and she was treated with oxygen, vasopressor drugs, diuretics, and assisted ventilation.

The blood count was normal, blood urea 48 $\mathrm{mg} / 100 \mathrm{ml}$, creatinine $\mathrm{r} \cdot 7 \mathrm{mg} / 100 \mathrm{ml}$, glucose $213 \mathrm{mg} / 100 \mathrm{ml}$. Electrolytes were normal. SGOT 80 units, LDH 250 units. Cholesterol $220 \mathrm{mg} /$ $100 \mathrm{ml}$.

Urine specific gravity ror3, albumin $4+$, glucose 0 , occasional erythrocytes.

The patient's general condition deteriorated and she died 8 hours after admission. Unfortunately permission for necropsy was denied.

An electrocardiogram showed deep $T$ wave inversion in leads II, III, and aVF suggestive of acute nontransmural diaphragmatic wall myocardial infarction. Occasional junctional premature beats were also present. A continuous strip of lead II is illustrated in the Fig., which shows a double set of $P$ waves: one set of $P$ waves of normal configuration at a rate of 75-80 a minute from the sinus node pacemaker and conducting to the ventricles; the second set of $\dot{P}$ waves at a rate of 40-45 a minute are somewhat sharp, peaked, and tend to be followed by microfibrillatory waves with a duration of 0.20 to $0.60 \mathrm{sec}$; these $P$ waves do not conduct to the ventricles and are from the ectopic pacemaker in the other atrium. The two rhythms do not interfere with each other. These findings are diagnostic of atrial dissociation. Depressed PR segment clearly seen in lead II is compatible with atrial ischaemia and/or infarction.

\section{Discussion}

Schrumpf in 1920 (Decherd, Ruskin, and Brindley, 1946) published the first electro- 
cardiogram illustrating a double set of $P$ waves in a 37-year-old man with rheumatic heart disease (mitral stenosis and aortic insufficiency).

Scherf and Cohen (1964) subsequently reported many similar cases, which they believed represented interatrial block. One of the cases was quite similar to that presented in this report: a 68-year-old woman was admitted with a diagnosis of acute myocardial infarction. The electrocardiogram revealed a double set of $\mathbf{P}$ waves and showed atrial dissociation. At necropsy occlusion of the left coronary artery was found above the origin of the atrial branch, which may have caused ischaemic damage to the interatrial conduction pathway resulting in atrial dissociation.

One of the cases of atrial dissociation reported by Chung, Walsh, and Massie (1965) proved to have atrial infarction at necropsy. A case of atrial dissociation reported by Abarquez and La Due (196I) was also attributed to atrial infarction. The cause of atrial dissociation in the present case may also have been atrial ischaemia and/or atrial infarction with ventricular myocardial infarction.

Atrial arrhythmias and atrial conduction disturbances are well known to occur after atrial infarction. In 17 per cent of 182 cases of ventricular myocardial infarction, atrial infarction was also proven at necropsy (Cushing et al., 1942). The incidence of atrial arrhythmias was not reported in this series.

Atrial dissociation has been found in many clinical conditions besides acute and chronic coronary thrombosis, such as digitalis toxicity, rheumatic heart disease, uraemia, hypertension, and diphtheria.

Three cases of atrial dissociation, two of these with unilateral atrial fibrillation, were reported by Deitz et al. (1957). Digitalis toxicity may well have been the cause of atrial dissociation in two cases. Strophanthin is known to produce dissociation of atrial fibres in toxic doses in animals.

Atrial dissociation has been observed in critically ill patients and occurs a few hours before death, as pointed out in previously reported cases, and confirmed by the present case. Hence the appearance of atrial dissociation carries a grave prognostic significance.

Mechanism It has been suggested that in atrial dissociation each atrium beats separately and independently as a result of blockage or interruption of an interatrial pathway (Bachmann's bundle). The sinus pacemaker controls one atrium and the ectopic pacemaker controls the other atrium. The two pacemakers do not interfere with each other as a result of

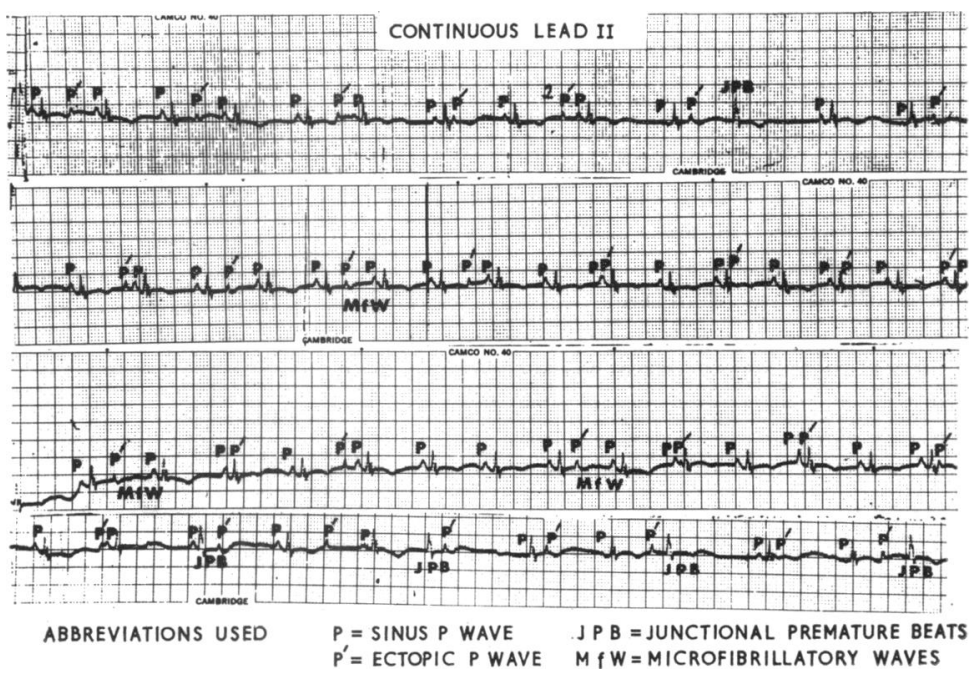

FIG. A continuous recording of lead II which demonstrates atrial dissociation. Inverted $T$ waves are present. Occasionally $P$ and $P^{\prime}$ waves fall on each other, giving rise to tall $P$ waves, pseudo-fusion beats, as the two $P$ waves do not really fuse because of interatrial block. Depressed PR segment is demonstrated and junctional premature beats are present.

interatrial block. $\mathbf{P}^{\prime}$ waves from the ectopic pacemaker do not conduct to the ventricles.

The phenomenon of atrial dissociation has recently been attributed to dissociation of the atrial myocardium with ectopic impulse formation and protective entrance and exit block.

Differential diagnosis Double sets of $P$ waves also occur in atrial parasystolic rhythm. The second set of $P$ waves in atrial parasystole will conduct to the ventricles whenever the ectopic $\mathbf{P}$ wave finds atrioventricular conduction pathways which are not refractory in contrast to the ectopic $P^{\prime}$ wave in atrial dissociation which never conducts to the ventricles. Moreover, the ectopic $\mathbf{P}$ waves in parasystole are more normal in appearance than the $P^{\prime}$ waves in atrial dissociation which tend to be peaked and somewhat bizarre.

The electrocardiogram findings after cardiac transplantation are identical to those observed in atrial dissociation. The rhythm originating from the donor's heart is the dominant rhythm and controls the ventricles, whereas rhythm originating from the recipient's heart only controls the part of atrial myocardium left behind after cardiac transplantation. This phenomenon results in two 
sets of $\mathbf{P}$ waves, and differential diagnosis from atrial dissociation may be impossible unless the history of cardiac transplantation is available.

As indicated by Scherf and Cohen (1964) and by Bellet (1963), some authors, including Katz (1946), consider the second set of $P$ waves as artefact and attribute them to contraction of the diaphragm, loose contact of electrodes, or electrical activity from the technician who has fortuitously made contact with an electrode, or to rhythmic skeletal muscle contractions. These points should be kept in mind before diagnosis of atrial dissociation is made, and special efforts should be undertaken to avoid any circumstances which may produce artefacts on the electrocardiogram.

I wish to thank Professor J. F. Goodwin who kindly reviewed this manuscript for me.

\section{References}

Abarquez, R. F., and La Due, J. S. (196I). Atrial dissociation. American fournal of Cardiology, 8, 448.
Bellet, S. (1963). Clinical Disorders of the Heart Beat, 2nd ed. pp. 431-434. Lea and Febiger, Philadelphia; Kimpton, London.

Chung, K.-Y., Walsh, T. J., and Massie, E. (1965). A review of atrial dissociation with illustrative cases and critical discussion. American fournal of the Medical Sciences, 250, 72.

Condorelli, L. (1929). Experimentelle Untersuchungen über die interaurikuläre Reizleitung. Zeitschrift für die gesamte experimentelle Medizin, 68, 516.

Cushing, E. H., Feil, H. S., Stanton, E. J., and Wartman, W. B. (1942). Infarction of the cardiac auricles (atria): clinical, pathological, and experimental studies. British Heart fournal, 4, 17.

Decherd, G. M., Jr., Ruskin, A., and Brindley, P. (1946). Interatrial and sinoatrial block. American Heart fournal, 31, 352.

Deitz, G. W., III, Marriot, H. J. L., Fletcher, E., and Bellet, S. (1957). Atrial dissociation and uniatrial fibrillation. Circulation, 15, 883.

Katz, L. N. (1946). Electrocardiography, 2nd ed., p. 772. Lea and Febiger, Philadelphia.

Scherf, D., and Cohen, J. (1964). The Atrioventricular Node and Selected Cardiac Arrhythmias, pp. 336364. Grune and Stratton, New York.

Requests for reprints to Dr. Abdul Hafiz Khan, Department of Medicine (Clinical Cardiology), Royal Postgraduate Medical School, Hammersmith Hospital, Du Cane Road, London W.r2. 